\title{
Mechanisms of energy-efficient magnetization switching in a bistable nanowire
}

\author{
M. H. A. Badarneh ${ }^{1}$, G. J. Kwiatkowski ${ }^{1}$, P. F. Bessarab ${ }^{1,2,3}$ \\ ${ }^{1}$ Science Institute, University of Iceland, 107 Reykjavík, Iceland \\ ${ }^{2}$ ITMO University, 197101 St. Petersburg, Russia \\ ${ }^{3}$ Peter Grünberg Institute and Institute for Advanced Simulation, \\ Forschungszentrum Jülich, 52425 Jülich, Germany \\ bessarab@hi.is
}

\begin{abstract}
Theoretical calculations of optimal control paths minimizing the energy cost of the magnetization reversal in 1D magnetic nanowires are presented. The energy-efficient reversal mechanism is studied as a function of the nanowire length and Gilbert damping parameter. For short nanowires, the optimal reversal mechanism corresponds to a uniform rotation of magnetization. If the length of the wire exceeds a certain critical length defined by the material parameters, switching time and damping, a standing spin wave emerges during magnetization switching. Comparison between the calculated optimal control paths and minimum energy paths reveals that realization of high energy efficiency of switching does not necessarily translate to the minimization of the energy barrier between the target magnetic states.
\end{abstract}

Keywords: magnetic nanowire, magnetization switching, optimal control.

Received: 8 May 2020

\section{Introduction}

Magnetic nanowires are characterized by an extended length and a nanoscale lateral size [1]. The high lengthto-width aspect ratio gives rise to large magnetic anisotropy, which usually results in two stable orientations of magnetization along the wire's axis. Bistability makes magnetic nanowires particularly promising as a basis of novel devices for data transmission, storage and processing where information can be encoded by domains with opposite magnetization [2]. Development of this technology depends critically on the knowledge about mechanisms of the magnetization reversal - a physical implementation of a bit operation. How reversal modes depend on the switching stimulus, magnetic parameters and geometry of the wire is also interesting from a fundamental point of view. Previous theoretical [3-9] and experimental [10-14] studies revealed three main mechanisms of magnetization switching in nanowires, including homogeneous rotation of magnetization and propagation of transverse and vortex domain walls. The possibility to achieve magnetization switching by following different transition mechanisms leads to a notion about the existence of a special mechanism that is optimal in terms of the termination time and energy cost. Definite identification of such optimal reversal mechanisms is particularly important for the development of energy-efficient information technologies based on magnetic nanowires.

In this article, we describe the application of the optimal control theory $[15,16]$ to the problem of energy-efficient magnetization reversal in a 1D magnetic nanowire. The theory makes it possible to identify optimal control paths (OCPs), i.e. reversal trajectories minimizing the energy cost of magnetization switching. We present numerical calculations of OCPs for various values of the nanowire length and Gilbert damping parameter. We report a crossover between the coherent rotation of magnetization and spin wave assisted switching. Finally, we compare calculated OCPs with the other distinguished paths in the configuration space - the minimum energy paths (MEPs).

The article is organized as follows. In Section 2, theoretical background and details of numerical calculations are described. Results of applications of the optimal control method are given in Section 3. A single macrospin test system is considered first. Then, the application to the magnetization reversal in a nanowire is described. Comparison between calculated OCPs and MEPs is also given. Summary and outlook are presented in Section 4. 


\section{Methods}

Magnetic nanowire is modeled as a 1D chain of $N$ magnetic moments extending along the $x$ axis, where each moment has the same magnitude $\mu$. The energy of the wire is given by a classical Heisenberg-type Hamiltonian:

$$
E=-J \sum_{i=1}^{N-1} \vec{s}_{i} \cdot \vec{s}_{i+1}-K \sum_{i=1}^{N}\left(\vec{s}_{i} \cdot \vec{e}^{x}\right)^{2},
$$

where $\vec{s}_{i}$ is the unit vector defining the orientation of the magnetic moment at site $i$. The first term in Eq. (1) describes the exchange interaction between nearest neighbours with ferromagnetic coupling constant $J>0$. The second term is due to magnetic uniaxial anisotropy characterized by the easy axis $\vec{e}^{x}$ along the wire and effective parameter $K>0$. Due to the anisotropy, there are two stable states in the system with moments aligned parallel or antiparallel to the $x$ axis, as illustrated in Fig. 1. The following values of the material parameters are used:

$$
\begin{aligned}
J & =562 \mathrm{meV}, \\
K & =37 \mathrm{meV}, \\
\mu & =4076 \mu_{B},
\end{aligned}
$$

where $\mu_{B}$ is Bohr magneton.

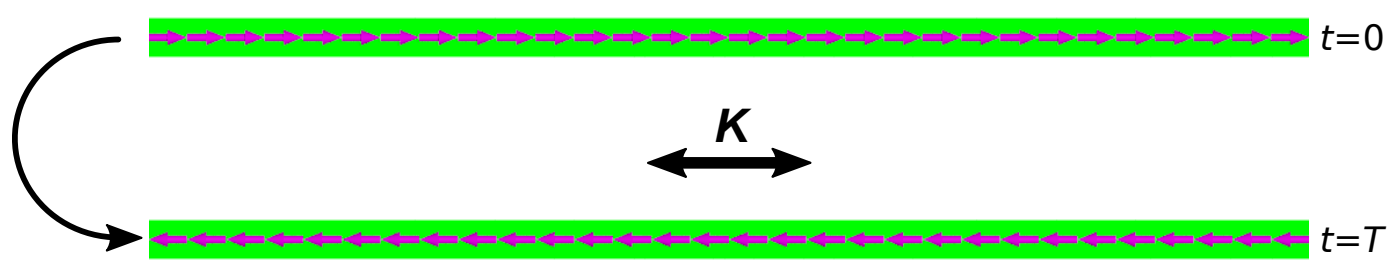

FIG. 1. Stable orientations of the magnetization in a nanowire. The nanowire is represented by a chain of $N=30$ magnetic moments. Arrows indicate orientation of the moments. Direction of the anisotropy axis is shown with the double-headed arrow. Transition between the states is schematically shown with the black arrow. In the OCP, the initial state is realized at $t=0$, while the final state is realized at $t=T$

We consider energy-efficient switching of magnetization in the wire from one stable orientation to the other. The switching process is induced by application of external magnetic field. The aim is to find the optimal field that realizes switching in a given time $T$ while minimizing the energy cost. Following Refs. $[15,16]$, we define the energy cost to be proportional to the amplitude of the applied magnetic field squared:

$$
\Phi=\int_{0}^{T} \sum_{j=1}^{N}\left|\vec{B}_{j}(t)\right|^{2} d t
$$

where $\vec{B}_{j}(t)$ is the applied magnetic field at site $j$ and time $t$. Summation over the sites where magnetic moments are localized is equivalent to spatial integration for a continuous system. The functional $\Phi$ needs to be minimized subject to the boundary conditions

$$
\begin{aligned}
s_{j}^{x}(t=0) & =1, \quad j=1, \ldots, N, \\
s_{j}^{x}(t=T) & =-1, \quad j=1, \ldots, N,
\end{aligned}
$$

and an equation of motion which is taken to be the Landau-Lifshitz-Gilbert equation:

$$
\left(1+\alpha^{2}\right) \dot{\vec{s}}_{j}=-\gamma \vec{s}_{j} \times\left(\vec{B}_{\mathrm{int}, j}+\vec{B}_{j}\right)-\alpha \gamma \vec{s}_{j} \times\left[\vec{s}_{j} \times\left(\vec{B}_{\mathrm{int}, j}+\vec{B}_{j}\right)\right],
$$

where $\alpha$ is the Gilbert damping factor, $\gamma$ is the gyromagnetic ratio, dot means time derivative and $\vec{B}_{\text {int }, j}$ is the internal magnetic field defined through the following equation:

$$
\vec{B}_{\mathrm{int}, j}=-\frac{1}{\mu} \frac{\partial E}{\partial \vec{s}_{j}},
$$

with internal energy $E$ given by Eq. (1). Equation (8) can be used to express $\vec{B}_{j}(t)$ in terms of the dynamical trajectory of the system as well as the magnetic field [16]:

$$
\vec{B}_{j}(t)=\frac{\alpha}{\gamma} \dot{\vec{s}}_{j}(t)+\frac{1}{\gamma}\left[\vec{s}_{j}(t) \times \dot{\vec{s}}_{j}(t)\right]-\vec{B}_{\mathrm{int}, j}^{\perp}(t) .
$$


Here, $\vec{B}_{\text {int }, j}^{\perp}(t)$ is a transverse component of $\vec{B}_{\text {int }, j}$ :

$$
\vec{B}_{\text {int }, j}^{\perp}=\vec{B}_{\text {int }, j}-\left(\vec{B}_{\text {int }, j} \cdot \vec{s}_{j}\right) \vec{s}_{j} .
$$

Upon substituting Eqs.(10)-(11) into Eq. (5), the energy cost $\Phi$ becomes a functional of $\vec{s}_{1}(t), \vec{s}_{2}(t), \ldots, \vec{s}_{N}(t)$. Before applying the variational principle to the functional $\Phi$, we discretize the time integral in Eq. (5) using the midpoint rule:

$$
\Phi\left[\vec{s}_{1}(t), \ldots, \vec{s}_{N}(t)\right] \approx \Phi(\mathbf{s})=\sum_{p=0}^{Q} \sum_{j=1}^{N} B_{p+\frac{1}{2}, j}^{2}\left(t_{p+1}-t_{p}\right),
$$

where $\left\{t_{p}\right\}$ is a partition of the time interval $[0, T]$ such that $0=t_{0}<t_{1}<\ldots<t_{Q}<t_{Q+1}=T$. In the current implementation, all time subintervals are the same, $t_{p+1}-t_{p}=\Delta t=T /(Q+1), p=0, \ldots, Q$. Trajectory of each magnetic moment, $\vec{s}_{j}(t)$, is then represented by a polygeodesic line connecting $Q+2$ images, $\vec{s}_{j}(t):\left\{\vec{s}_{0, j}, \ldots, \vec{s}_{Q+1, j}\right\}$, with $\vec{s}_{p, j}=\vec{s}_{j}\left(t_{p}\right)$, where the end points are fixed according to the boundary conditions [see Eqs. (6)-(7)], but $Q$ intermediate images need to be adjusted to an optimal configuration representing the minimum of $\Phi$. The functional $\Phi$ is turned into a $3 Q N$-dimensional function of image positions, $\Phi=\Phi(\mathbf{s})$, with $\mathbf{s}=\left\{\vec{s}_{1,1}, \vec{s}_{1,2}, \ldots, \vec{s}_{1, N}, \vec{s}_{2,1}, \ldots, \vec{s}_{Q, N}\right\}$. The sought-for optimal control path (OCP) minimizing the energy cost of switching is found by choosing some initial guess for the position of the images and then bringing that to a local minimum of $\Phi(\mathbf{s})$ by means of the (limited-memory) Broyden-Fletcher-Goldfarb-Shanno algorithm [17] adapted to magnetic degrees of freedom $[18,19]$. The final, relaxed configuration of the images gives a discrete representation of the OCP. The optimal external field pulse is then obtained from the OCP using Eq. (10).

\section{Results and discussion}

\subsection{Test problem}

The numerical method described earlier is first illustrated with calculations of the OCP for the reversal of a single magnetic moment whose internal energy is defined by the anisotropy along $z$ axis,

$$
E_{1}=-K\left(\vec{s} \cdot \vec{e}^{z}\right)^{2} \text {. }
$$

The value of the anisotropy constant $K$ is defined in Eq. (3). The OCP between the energy minima at $s^{z}= \pm 1$ for this system can be obtained analytically in terms of elliptic functions [16], providing a benchmark for numerical calculations. Discrete representations of the OCP were obtained numerically by relaxing $Q$ movable images to a local minimum of the energy cost $\Phi$. The calculations were considered converged when the magnitude of the gradient of $\Phi$ had dropped by twelve orders of magnitude.
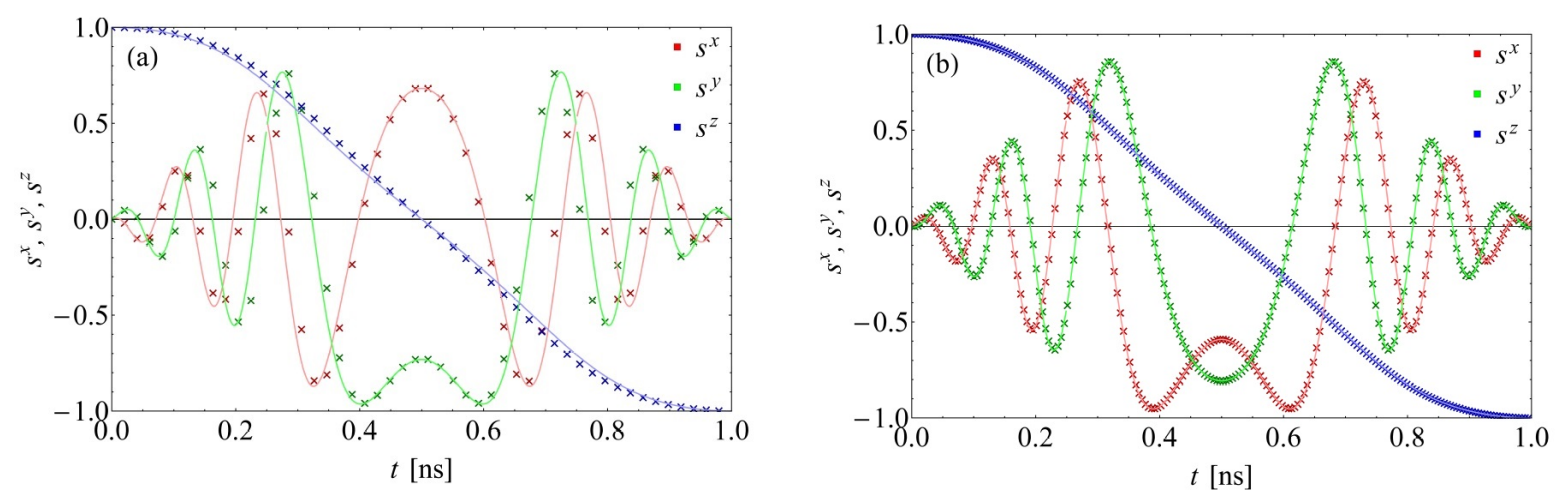

FIG. 2. Evolution of the magnetic moment along the OCP for switching time $T=1 \mathrm{~ns}$ and damping parameter $\alpha=0.1$. Analytical solution is given by solid lines, position of images included in the numerical calculations is shown with crosses. $Q=50$ (a) and $Q=200$ (b) movable images were used in the numerical OCP calculations

Figure 2 shows comparison between the analytical solution and numerical results obtained for $Q=50$ and $Q=200$ images. Both analytical and numerical calculations were performed for $\alpha=0.1$ and $T=1 \mathrm{~ns}$. Agreement between the analytical and numerical results is better for larger number of images, as expected. We have found that using $Q \approx 200$ movable images in the OCP calculations was sufficient to ensure relative error to be below $1 \%$. 

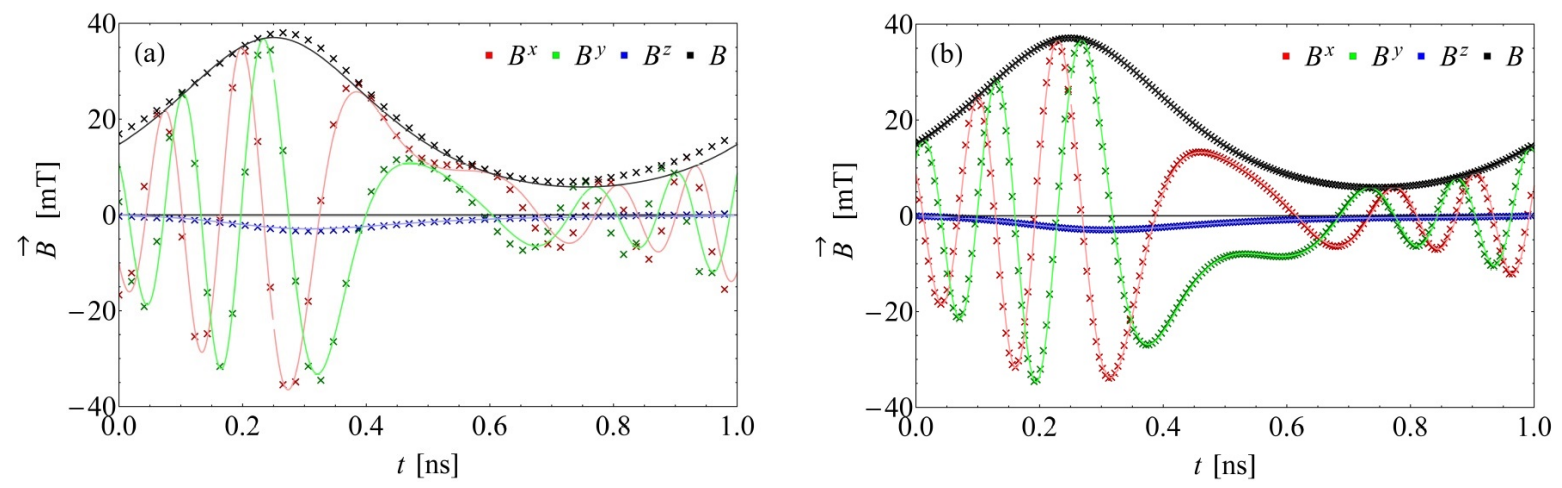

FIG. 3. Optimal switching field derived from the OCP shown in Fig. 2. Switching time $T=1 \mathrm{~ns}$ and damping parameter $\alpha=0.1$. Analytical solution is given by solid lines, crosses correspond to position of images included in the numerical OCP calculations. $Q=50$ (a) and $Q=200$ (b) movable images were used in the OCP calculations

The calculated OCP describes a steady motion of the magnetic moment from the initial state at $s^{z}=1$ to the final state at $s^{z}=-1$. The monotonic decrease in $s^{z}$ is accompanied by a precessional motion around the anisotropy axis. Note that the precession reverses its direction upon reaching the top of the energy barrier at $t=T / 2$. Fig. 3 shows how the optimal switching field derived from the OCP depends on time. Direction of the calculated optimal field is synchronized with the motion of the magnetic moment so that it is orthogonal to $\vec{s}$ at any time. Moreover, $\vec{B}(t)$ is modulated in amplitude. The internal torque arising due to damping counteracts the switching motion before crossing the energy barrier. Therefore, overall larger field needs to be applied in the first half of the reversal process compared to that in the second half, where the damping torque aids switching. A careful analysis demonstrates that $B(t)$ reaches a maximum at $t=T / 4$ and a minimum at $t=3 T / 4$, which coincides with the position of the extrema of the polar component of the internal torque.

\subsection{Magnetization switching in a nanowire}

Fig. 4 illustrates mechanisms of energy efficient magnetization reversal in the nanowire revealed by the OCP calculations. Possible mechanisms include uniform rotation of magnetization [see Fig. 4(a)] and emergence of a standing spin wave with variable wavelength [see Fig. 4(b,c)]. In the case of the coherent rotation mechanism, each magnetic moment in the wire follows the same trajectory presented in the previous subsection.

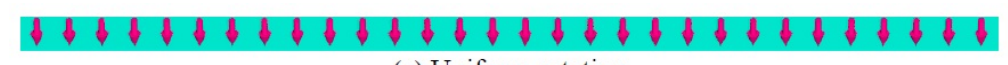

(a) Uniform rotation

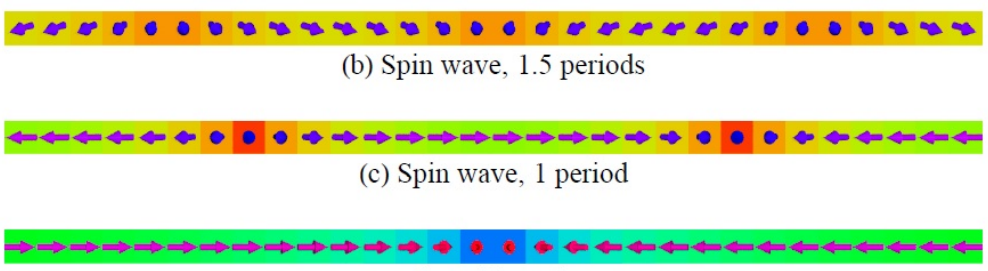

(d) Saddle point

FIG. 4. Magnetic configurations realized along OCPs (a)-(c) and MEP (d) for magnetization switching in the nanowire of length $N=30$. For the OCPs, configurations at $t=T / 2$ are shown where $T=1 \mathrm{~ns}$. For the MEP, the saddle point configuration is presented. OCP corresponds to a coherent rotation of magnetic moments for $\alpha=0.1$ (a) and to spin wave assisted switching with 1.5 wave periods for $\alpha=0.5$ (b) and with 1 wave period for $\alpha=0.6$ (c). MEP corresponds to a transient domain wall nucleation and propagation $(\mathrm{d})$

How the result of the OCP calculations depend on the parameters of the nanowire is illustrated in Fig. 5. Specifically, Fig. 5(a) shows the calculated energy cost $\Phi$ of optimal switching as a function of the length $N$ of the wire for the fixed values of the switching time and damping factor with $T=1 \mathrm{~ns}$ and $\alpha=0.4$. For relatively short nanowires, $\Phi$ 
scales linearly with the wire length which is a consequence of the uniform rotation of the magnetization. At $N \approx 60$, a salient point is evident in the $\Phi(N)$ dependence as a result of the crossover to spin-wave assisted switching. As the number of magnetic moments in the wire increases, progressively more periods of the spin wave is observed. Note that the number of the spin-wave periods can assume both half-integer and integer values [see Fig. 4(b,c)].
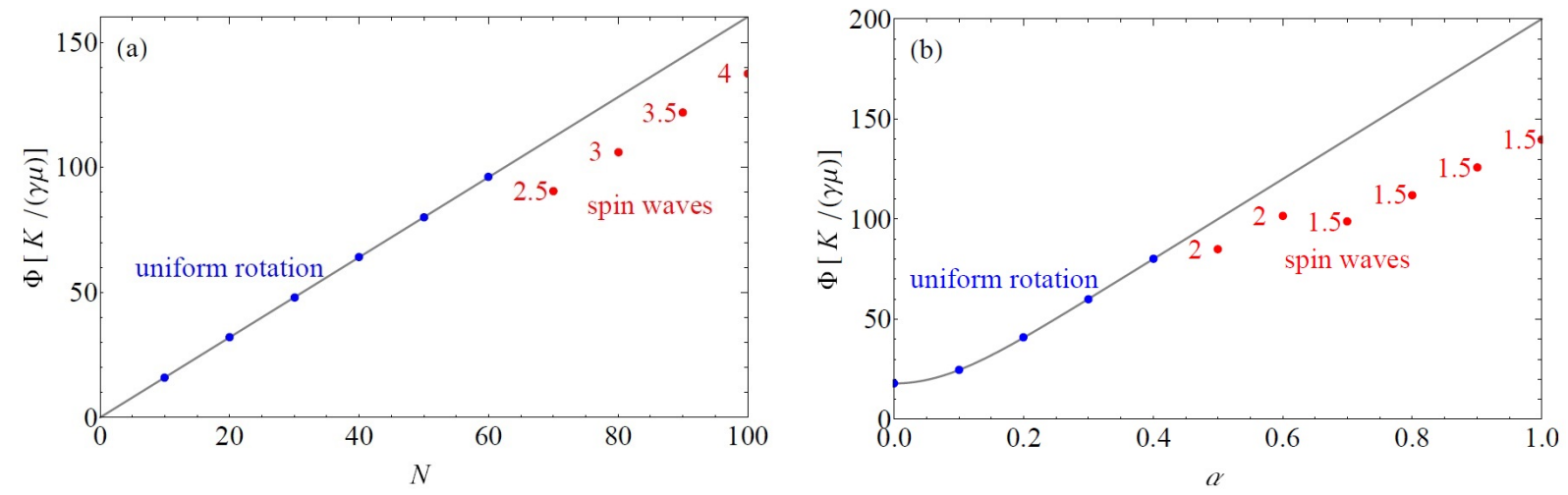

FIG. 5. Minimum energy cost of magnetization switching as a function of the nanowire length $N$ for $\alpha=0.4$ and $T=1 \mathrm{~ns}$ (a) and damping parameter $\alpha$ for $N=50$ and $T=1 \mathrm{~ns}$ (b). Uniform rotation and spin wave solutions are shown with blue and red circles, respectively. Red labels indicate the number of wavelengths in the spin-wave solutions. Solution for the macrospin approximation is shown with a gray line

Interestingly, the mechanism of the energy-efficient magnetization switching depends on the damping parameter, as illustrated in Fig. 5(b) showing $\Phi$ as a function of $\alpha$ for $N=50$ and $T=1$ ns. When $\alpha \leq 0.4$, the optimal switching mechanism corresponds to the uniform rotation of magnetization and $\Phi(\alpha)$ follows the dependence predicted in Ref. [16]. As the damping factor increases, the switching mechanism changes to the spin-wave, first with 2 periods and then with 1.5 periods. Each crossover event is accompanied by a sharp change in the slope of the $\Phi(\alpha)$ function.

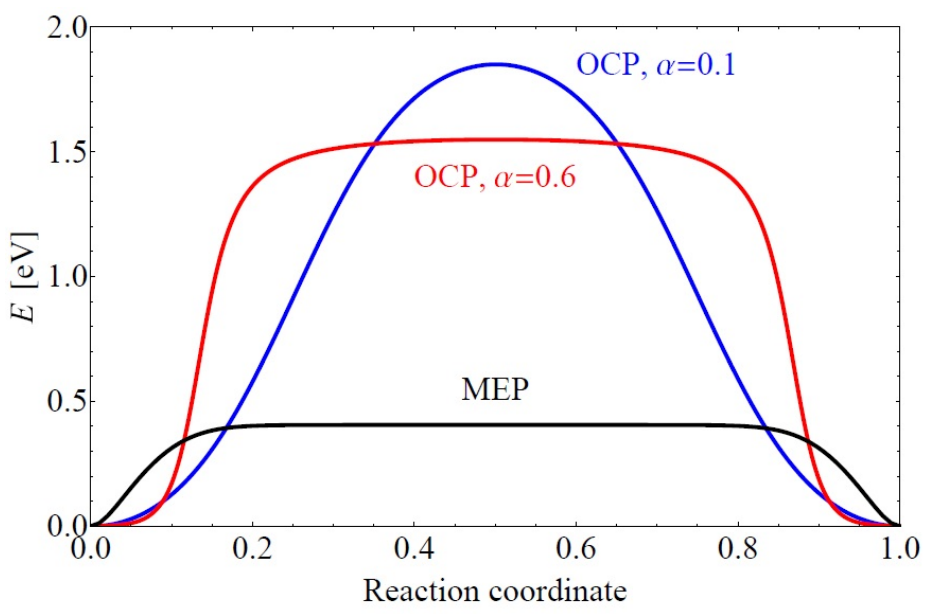

FIG. 6. Variation of the energy of the nanowire with $N=50$ along the MEP (black line) and the OCP for $T=1 \mathrm{~ns}, \alpha=0.1$ (blue line) and $\alpha=0.6$ (red line). Reaction coordinate is defined as a normalized displacement along the path

It is informative to compare the calculated OCPs with the other distinguished paths in the configuration space minimum energy paths (MEPs). An MEP connecting two stable states is a path lying lowermost on the energy surface, and the point of the highest energy along the MEP - a saddle point on the energy surface - defines the energy barrier between the states, the primary quantity determining their thermal stability within harmonic rate theories [20-22]. The MEPs for the magnetization reversal in the nanowires considered here were calculated using the geodesic nudged elastic band method [23]. The MEP changes from the uniform rotation to the nucleation and propagation of a transient 
domain wall when the length of the wire exceeds the domain wall width. The saddle-point configuration for the nanowire with $N=30$ is shown in Fig. 4(d) as an example. These paths are very different from the calculated OCPs. In fact, the OCP calculations have never converged on the path corresponding to the single domain wall propagation, even if the initial guess was set to be the MEP. Note that an MEP is completely defined by the energy surface of the system, and, therefore, is independent of dynamical properties such as the switching time or damping parameter. In contrast, an OCP is a valid dynamical trajectory, which in particular results in that the mechanism of energy-efficient magnetization reversal depends on $\alpha$, as demonstrated above.

Finally, the energy variations along the calculated MEP and OCPs are compared in Fig. 6 for the nanowire with $N=50$. The energy profile along the MEP is flat, which is a typical feature for the domain wall propagation costing almost no energy. The energy barrier derived from the MEP is much smaller than the highest energy point along the OCPs. This result suggests a conclusion that optimal control of magnetization switching which minimizes the energy cost does not necessarily translates into the minimization of the energy barrier between the target states. Following an OCP involves rotation of magnetic moments in such a way that the influence of the external stimulus is minimized, but the system's internal dynamics is effectively used to aid magnetization switching.

\section{Conclusion}

In conclusion, we have studied energy-efficient magnetization reversal in bistable magnetic nanowires by means of OCP calculations. The calculations demonstrate that short nanowires reverse their magnetization via coherent rotation that combines a steady advancement toward the target state and precession around the anisotropy axis. If the length of the wire exceeds a certain critical length, a standing spin wave emerges during magnetization switching. In contrast to MEPs, OCPs demonstrate dependence on dynamical parameters such as switching time and damping. Our results deepen the understanding of the optimal control of magnetization switching in nanowires.

\section{Acknowledgements}

The authors would like to thank T. Sigurjónsdóttir for helpful discussions. This work was funded by the Russian Science Foundation (Grant No. 19-72-10138), the Icelandic Research Fund (Grant No. 184949-052), and the Alexander von Humboldt Foundation.

\section{References}

[1] Staňo M., Fruchart O. Magnetic nanowires and nanotubes. In Handbook of Magnetic Materials, vol. 27, ed. Brück E. Elsevier, Amsterdam., 2018, P. 155-267.

[2] Parkin S. S. P., Hayashi M. Thomas L. Magnetic domain-wall racetrack memory. Science, 2008, 320(5873), P. 190-194.

[3] Hinzke D., Nowak U. Magnetization switching in nanowires: Monte Carlo study with fast Fourier transformation for dipolar fields. Journal of Magnetism and Magnetic Materials, 2000, 221, P. 365-372.

[4] Hertel R., Kirschner J. Magnetization reversal dynamics in nickel nanowires. Physica B, 2004, 343, P. $206-210$.

[5] Allende S., Altbir D., Salcedo E., Bahiana M., Sinnecker J. P. Propagation of transverse domain walls in homogeneous magnetic nanowires. Journal of Applied Physics, 2008, 104, P. 013907.

[6] Ivanov Yu. P., Vázquez M., Chubykalo-Fesenko O. Magnetic reversal modes in cylindrical nanowires. Journal of Physics D: Applied Physics, 2013, 46, P. 485001

[7] Goussev A., Lund R. G., Robbins J. M., Slastikov V., Sonnenberg C. Domain wall motion in magnetic nanowires: an asymptotic approach. Proceedings of the Royal Society A, 2013, 469, P. 20130308.

[8] Sultan M.S., Atkinson D. Aspect-ratio dependence of magnetization reversal in cylindrical ferromagnetic nanowires. Materials Research Express, 2016, 3, P. 056104.

[9] Kuncser A., Antohe S., Kuncser, V. A general perspective on the magnetization reversal in cylindrical soft magnetic nanowires with dominant shape anisotropy. Journal of Magnetism and Magnetic Materials, 2017, 423, P. 34-38.

[10] Endo Y., Fujimoto H., Kumano S., Matsumura Y., Sasaki I., Kawamura Y., Yamamoto M., Nakatani R. Study on the magnetization reversal process in a magnetic nanowire and a magnetic dot observed by magnetic field sweeping magnetic force microscopy measurements. Journal of Applied Physics, 2008, 103, P. 07D918.

[11] Lavín R., Denardin J. C., Escrig J., Altbir D., Cortés A., Gómez H. Angular dependence of magnetic properties in Ni nanowire arrays. Journal of Applied Physics, 2009, 106, P. 103903.

[12] Vivas L. G., Vazquez M., Escrig J., Allende S., Altbir D., Leitao D. C., Araujo J. P. Magnetic anisotropy in CoNi nanowire arrays: Analytical calculations and experiments. Physical Review B, 2012, 85, P. 035439.

[13] García J., Prida V. M., Vivas L. G., Hernando B., Barriga-Castro E. D., Mendoza-Reséndez R., Luna C., Escrig J., Vázquez M. Magnetization reversal dependence on effective magnetic anisotropy in electroplated Co-Cu nanowire arrays. Journal of Materials Chemistry C, 2015, 3, P. 4688.

[14] Sultan M. S. Angular dependence of switching behaviour in template released isolated NiFe nanowires. Physics Letters A, 2017, 381, P. 38963903.

[15] Barros N., Rassam M., Jirari H., Kachkachi H. Optimal switching of a nanomagnet assisted by microwaves. Physical Review B, 2011, 83, P. 144418 
[16] Kwiatkowski G. J., Badarneh M. H. A., Berkov D. V., Bessarab P. F. Optimal control of magnetization reversal in a monodomain particle by means of applied magnetic field, 2020. arXiv: https://arxiv.org/abs/2004.02146.

[17] Nocedal J., Wright S. J. Numerical Optimization (2nd ed.). Springer, New York, 2006.

[18] Ivanov A. V., Uzdin V. M., Jónsson H. Fast and robust algorithm for the energy minimization of spin systems applied in an analysis of high temperature spin configurations in terms of skyrmion density, 2019. arXiv: https://arxiv.org/abs/1904.02669.

[19] Ivanov A. V., Dagbjartsson D., Tranchida J., Uzdin V. M., Jónsson H. Efficient optimization method for finding minimum energy paths of magnetic transitions. Journal of Physics: Condensed Matter, 2020. Accepted manuscript. URL: https://doi.org/10.1088/1361-648X/ab8b9c.

[20] Kramers H. Brownian motion in a field of force and the diffusion model of chemical reactions. Physica. 1940. 7. P. $284-304$.

[21] Vineyard G. H. Frequency factors and isotope effects in solid state rate processes. Journal of Physics and Chemistry of Solids, 1957, 3, P. 121-127.

[22] W. F. Brown W. F. Thermal fluctuation of fine ferromagnetic particles. IEEE Transactions on Magnetics, 1979, MAG-15, P. $1196-1208$.

[23] Bessarab P. F., Uzdin V. M., J'onsson H. Method for finding mechanism and activation energy of magnetic transitions, applied to skyrmion and antivortex annihilation. Computer Physics Communications, 2015, 196, P. 335-347. 